\title{
Modelling supply risks in interdependent manufacturing systems: A case study
}

\author{
Omega, R.S. , Noel, V.M. , Masbad, J.G. ${ }^{a}$, Ocampo, L.A. ${ }^{\text {b, }}$ \\ ${ }^{a}$ Department of Industrial Engineering, University of San Carlos, Cebu City, Philippines \\ ${ }^{b}$ Department of Mechanical and Manufacturing Engineering, University of San Carlos, Cebu City, Philippines
}

\section{A B S T R A C T}

This paper proposes a supply-driven inoperability input-output model (SIIM) in analysing risks of manufacturing systems. The approach, derived from the Leontief's input-output model, was previously debated for its implausibility in analysing sectors in an economic system. This paper provides interesting insights in production risk analysis especially that the adoption of SIIM in micro-level systems particularly in manufacturing systems was not yet explored in the current literature. The resemblance of economic systems and manufacturing systems in terms of system components, input-output concept, and component-wise interdependencies makes the approach appealing and highly plausible. Thus, this work adopts SIIM in analysing the impact of supply perturbations in a manufacturing system brought about by natural and man-made disasters, economic shifts, and government policies. An actual case study was carried out in a manufacturing firm in the central Philippines and two scenarios were presented to illustrate the proposed approach. The proposed approach is highly significant for manufacturing and risk practitioners in formulating mitigation policies to achieve a resilient manufacturing system.
\end{abstract}

\section{ARTICLE INFO}

Keywords:

Manufacturing systems

Supply chain

Supply risk analysis

Modelling

Supply-driven inoperability

Input-output model

*Corresponding author: laocampo@usc.edu.ph (Ocampo, Lanndon A.)

Article history:

Received 17 January 2016

Revised 16 May 2016

Accepted 23 May 2016

\section{References}

[1] Tan, C.S., Tan, P.S., Lee, S.S.G., Pham, M.T. (2013). An inoperability input-output model (IIM) for disruption propagation analysis, Proceedings of the 2013 IEEE International Conference on Industrial Engineering and Engineering Management (IEEM), 186-190, doi: 10.1109/IEEM.2013.6962400.

[2] Santos, J.R., Haimes, Y.Y. (2004). Modeling the demand reduction input-output (I-0) inoperability due to terrorism of interconnected infrastructures, Risk Analysis, Vol. 24, No. 6, 1437-1451, doi: 10.1111/j.0272-4332.2004. 00540.x.

[3] Jiang P., Haimes, Y.Y. (2004). Risk management for Leontief-based interdependent systems, Risk Analysis, Vol. 24, No. 5, 1215-1229, doi: 10.1111/j.0272-4332.2004.00520.x.

[4] Haimes, Y.Y., Horowitz, B.M., Lambert, J.H., Santos, J.R., Lian, C., Crowther, K.G. (2005). Inoperability input-output model for interdependent infrastructure sectors: Theory and methodology, Journal of Infrastructure Systems, Vol. 11, No. 2, 67-79, doi: 10.1061/(ASCE)1076-0342(2005)11:2(67).

[5] Santos, J.R. (2006). Inoperability input-output modeling of disruptions to interdependent economic systems, Systems Engineering, Vol. 9, No. 1, 20-34, doi: 10.1002/sys.20040.

[6] Leung, M., Haimes, Y.Y., Santos, J.R. (2007). Supply-and output-side extensions to the inoperability input-output model for interdependent infrastructures, Journal of Infrastructure Systems, Vol. 13, No. 4, 299-310, doi: 10.1061/(ASCE)1076-0342(2007)13:4(299).

[7] Anderson, C.W., Santos, J.R., Haimes, Y.Y. (2007). A risk-based input-output methodology for measuring the effects of the August 2003 Northeast blackout, Economic Systems Research, Vol. 19, No. 2, 183-204, doi: $10.1080 / 09535310701330233$.

[8] Zobel, C.W. (2011). Representing perceived tradeoffs in defining disaster resilience, Decision Support Systems, Vol. 50, No. 2, 394-403, doi: 10.1016/i.dss.2010.10.001. 
[9] Wang, S., Hong, L., Chen, X. (2012). Vulnerability analysis of interdependent infrastructure systems: A methodological framework, Physica A: Statistical Mechanics and its Applications, Vol. 391, No. 11, 3323-3335, doi: 10.1016/j.physa.2011.12.043.

[10] Lei, T., Liangyu, W., Rijia, D., Lijia, L. (2011). Study on the dynamic input-output model with coal mine safety, Procedia Engineering, Vol. 26, 1997-2002, doi: 10.1016/i.proeng.2011.11.2396.

[11] Jiang, W., Deng, L., Chen, L., Wu, J., Li, J. (2009). Risk assessment and validation of flood disaster based on fuzzy mathematics, Progress in Natural Science, Vol. 19, No. 10, 1419-1425, doi: 10.1016/j.pnsc.2008.12.010.

[12] Miller, R.E., Blair, P.D. (2009). Input-output analysis: Foundations and extensions, (2 ${ }^{\text {nd }}$ edition), Cambridge University Press, New York, USA, doi: 10.1017/CB09780511626982.

[13] Jung, J., Santos, J.R., Haimes, Y.Y. (2009). International trade inoperability input-output model (IT-IIM): Theory and application, Risk Analysis, Vol. 29, No. 1, 137-154, doi: 10.1111/j.1539-6924.2008.01126.X.

[14] Tan, R.R., Aviso, K.B., Promentilla, M.A.B., Yu, K.D.S., Santos, J.R. (2015). Development of a fuzzy linear programming model for allocation of inoperability in economic sectors due to loss of natural resource inputs, DLSU Business \& Economics Review, Vol. 24, No. 2, 1-12.

[15] Tan, R.R., Aviso, K.B., Promentilla, M.A.B., Yu, K.D.S., Santos, J.R. (2014). Fuzzy inoperability input-output analysis of mandatory biodiesel blending programs: The Philippine case, Energy Procedia, Vol. 61, 45-48, doi: 10.1016/i.egypro.2014.11.902.

[16] Pant, R., Barker, K., Grant, F.H., Landers, T.L. (2011). Interdependent impacts of inoperability at multi-modal transportation container terminals, Transportation Research Part E: Logistics and Transportation Review, Vol. 47, No. 5, 722-737, doi: 10.1016/i.tre.2011.02.009.

[17] Resurreccion, J.Z., Santos, J.R. (2012). Stochastic modeling of manufacturing-based interdependent inventory for formulating sector prioritization strategies in reinforcing disaster preparedness, In: Proceedings of the 2012 IEEE Systems and Information Design Symposium (SIEDS), 134-139, doi: 10.1109/SIEDS.2012.6215127.

[18] Tan, R.R., Aviso, K.B., Promentilla, M.A.B., Solis, F.D.B., Yu, K.D.S., Santos, J.R. (2015). A shock absorption index for inoperability input-output models, Economic Systems Research, Vol. 27, No. 1, 43-59, doi: 10.1080/0953 5314. 2014.922462.

[19] Owusu, A., Mohamed, S., Anissimov, Y. (2010). Input-output impact risk propagation in critical infrastructure interdependency, In: Proceedings of the $13^{\text {th }}$ International Conference on Computing in Civil and Building Engineering, Nottingham, UK.

[20] Setola, R., De Porcellinis, S., Sforna, M. (2009). Critical infrastructure dependency assessment using the inputoutput inoperability model, International Journal of Critical Infrastructure Protection, Vol. 2, No. 4, 170-178, doi: 10.1016/j.ijcip.2009.09.002.

[21] Percoco, M. (2006). A note on the inoperability input-output model, Risk Analysis, Vol. 26, No. 3, 589-594, doi: 10.1111/j.1539-6924.2006.00765.x.

[22] Wei, H., Dong, M., Sun, S. (2010). Inoperability input-output modeling (IIM) of disruptions to supply chain networks, Systems Engineering, Vol. 13, No. 4, 324-339, doi: 10.1002/sys.20153.

[23] Barker, K., Santos, J.R. (2010). Measuring the efficacy of inventory with a dynamic input-output model, International Journal of Production Economics, Vol. 126, No. 1, 130-143, doi: 10.1016/j.ijpe.2009.08.011.

[24] Benjamin, M.F.D., Tan, R.R., Razon, L.F. (2015). Probabilistic multi-disruption risk analysis in bioenergy parks via physical input-output modeling and analytic hierarchy process, Sustainable Production and Consumption, Vol. 1, 22-33, doi: 10.1016/i.spc.2015.05.001.

[25] Garvey, P.R., Pinto, C.A., Santos, J.R. (2014). Modelling and measuring the operability of interdependent systems and systems of systems: advances in methods and applications, International Journal of System of Systems Engineering, Vol. 5, No. 1, 1-24, doi: 10.1504/IJSSE.2014.060880.

[26] Percoco, M. (2011). On the local sensitivity analysis of the inoperability input-output model, Risk Analysis, Vol. 31, No. 7, 1038-1042, doi: 10.1111/j.1539-6924.2010.01574.x.

[27] Oliva, G., Panzieri, S., Setola, R. (2010). Agent-based input-output interdependency model, International Journal of Critical Infrastructure Protection, Vol. 3, No. 2, 76-82, doi: 10.1016/j.ijcip.2010.05.001.

[28] Crowther, K.G. (2008). Decentralized risk management for strategic preparedness of critical infrastructure through decomposition of the inoperability input-output model, International Journal of Critical Infrastructure Protection, Vol. 1, 53-67, doi: 10.1016/i.ijcip.2008.08.009.

[29] Hester, P.T., Adams, K.MacG. (2013). Determining stakeholder influence using input-output modeling, Procedia Computer Science, Vol. 20, 337-341, doi: 10.1016/i.procs.2013.09.282.

[30] Lian, C., Santos, J.R., Haimes, Y.Y. (2007). Extreme risk analysis of interdependent economic and infrastructure sectors, Risk Analysis, Vol. 27, No. 4, 1053-1064, doi: 10.1111/j.1539-6924.2007.00943.x.

[31] Xu, W., Wang, Z. (2015). The uncertainty and sensitivity analysis of the interdependent infrastructure sectors based on the supply-driven inoperability input-output model, International Journal of Innovative Computing, Information and Control, Vol. 11, No. 2, 615-625.

[32] Whiteman, H. (2014). Philippines gets more than its share of disasters, from http://edition.cnn.com/ 2013/11/08/world/asia/philippines-typhoon-destruction/_accessed January 4, 2016.

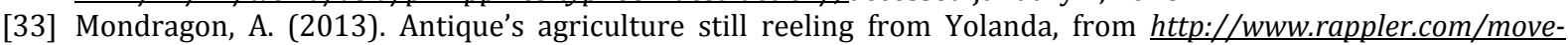
ph/46911-antique-agriculture-yolanda, accessed January 6, 2016.

[34] Olchondra, R.T., Abadilla, D.D. (2015). 5 dead, 4 missing in coal mine collapse. Inquirer News, from http://newsinfo.inquirer.net/705980/5-dead-4-missing-in-coal-mine-collapse accessed January 6, 2016.

[35] Li, M., Wu, G.-D., Lai, X.D. (2014). Capacity coordination mechanism for supply chain under supply-demand uncertainty, International Journal of Simulation Modelling, Vol. 13, No. 3, 364-376, doi: 10.2507/IJSIMM13(3)C014.

[36] Seuring, S. (2013). A review of modeling approaches for sustainable supply chain management, Decision Support Systems, Vol. 54, No. 4, 1513-1520, doi: 10.1016/j.dss.2012.05.053. 
[37] Smew, W., Young, P., Geraghty, J. (2013). Supply chain analysis using simulation, Gaussian process modelling and optimisation, International Journal of Simulation Modelling, Vol. 12, No. 3, 178-189, doi: 10.2507/IJSIMM12 (3)4.239.

[38] Očevčić, H., Nenadić, K., Šolić, K. (2014). Decision support based on the risk assessment of information systems and Bayesian learning, Tehnički vjesnik - Technical Gazette, Vol. 21, No. 3, 539-544. 


\title{
APEM
}

\section{Modeliranje tveganja oskrbovalnih verig v medsebojno odvisnih proizvodnih sistemih: študija primera}

\author{
Omega, R.S. , Noel, V.M. , Masbad, J.G. ${ }^{a}$, Ocampo, L.A. ${ }^{\text {b, }}$ \\ ${ }^{a}$ Department of Industrial Engineering, University of San Carlos, Cebu City, Philippines \\ ${ }^{\mathrm{b}}$ Department of Mechanical and Manufacturing Engineering, University of San Carlos, Cebu City, Philippines
}

\begin{abstract}
POVZETEK
$\mathrm{Za}$ analizo tveganja $\mathrm{v}$ proizvodnih sistemih predlaga prispevek vhodnoizhodni model (angl. SIIM), ki temelji na neoperabilnosti zaradi oskrbovanja. Pristop, ki izvira iz Leontiefovega vhodno-izhodnega modela, je doslej veljal kot neprepričljiv pri analiziranju problemov v ekonomskih sistemih. Ta prispevek ponuja zanimiv vpogled $v$ analizo proizvodnega tveganja, saj prilagoditev SIIM v mikronivojske sisteme v proizvodnih sistemih še ni bila raziskana. Vendar pa podobnost ekonomskih in proizvodnih sistemov v smislu sistemskih komponent, vhodno-izhodnih konceptov in medsebojne odvisnosti komponent dela pristop mikaven in prepričljiv. Pričujoča raziskava izkorišča SIIM pri analiziranju vpliva oskrbovalnih motenj v proizvodnih sistemih, ki so lahko posledica naravnih nesreč, nesreč zaradi človeškega faktorja, ekonomskih premikov in politike vlade. Za prikaz delovanja predlaganega pristopa je bila izvedena študija primera $\mathrm{z}$ dvema mogočima scenarijema v izbranem proizvodnem sistemu na Filipinih. Predlagan pristop je zelo pomemben za proizvodnjo in osebje, ki se ukvarja s tveganjem, saj omogoča ublažitev negativnih vplivov in prožen odziv proizvodnih sistemov.
\end{abstract}

\section{PODATKI O ČLANKU}

Ključne besede:

Izdelovalni sistemi

Oskrbovalna veriga

Tveganje v oskrbovalni verigi

Modeliranje

Neoperabilnost zaradi oskrbovanja

Vhodno-izhodni model

*Kontaktna oseba: laocampo@usc.edu.ph

(Ocampo, Lanndon A.)

Zgodovina članka:

Prejet 17. januarja 2016

Popravljen 16. maja 2016

Sprejet 23. maja 2016 


\section{References}

[1] Tan, C.S., Tan, P.S., Lee, S.S.G., Pham, M.T. (2013). An inoperability input-output model (IIM) for disruption propagation analysis, Proceedings of the 2013 IEEE International Conference on Industrial Engineering and Engineering Management (IEEM), 186-190, doi: 10.1109/IEEM.2013.6962400.

[2] Santos, J.R., Haimes, Y.Y. (2004). Modeling the demand reduction input-output (I-O) inoperability due to terrorism of interconnected infrastructures, Risk Analysis, Vol. 24, No. 6, 1437-1451, doi: 10.1111/j.0272-4332.2004. 00540.x.

[3] Jiang P., Haimes, Y.Y. (2004). Risk management for Leontief-based interdependent systems, Risk Analysis, Vol. 24, No. 5, 1215-1229, doi: 10.1111/i.0272-4332.2004.00520.x.

[4] Haimes, Y.Y., Horowitz, B.M., Lambert, J.H., Santos, J.R., Lian, C., Crowther, K.G. (2005). Inoperability input-output model for interdependent infrastructure sectors: Theory and methodology, Journal of Infrastructure Systems, Vol. 11, No. 2, 67-79, doi: 10.1061/(ASCE)1076-0342(2005)11:2(67).

[5] Santos, J.R. (2006). Inoperability input-output modeling of disruptions to interdependent economic systems, Systems Engineering, Vol. 9, No. 1, 20-34, doi: 10.1002/sys.20040.

[6] Leung, M., Haimes, Y.Y., Santos, J.R. (2007). Supply-and output-side extensions to the inoperability input-output model for interdependent infrastructures, Journal of Infrastructure Systems, Vol. 13, No. 4, 299-310, doi: 10.1061/(ASCE)1076-0342(2007)13:4(299).

[7] Anderson, C.W., Santos, J.R., Haimes, Y.Y. (2007). A risk-based input-output methodology for measuring the effects of the August 2003 Northeast blackout, Economic Systems Research, Vol. 19, No. 2, 183-204, doi: $10.1080 / 09535310701330233$.

[8] Zobel, C.W. (2011). Representing perceived tradeoffs in defining disaster resilience, Decision Support Systems, Vol. 50, No. 2, 394-403, doi: 10.1016/i.dss.2010.10.001.

[9] Wang, S., Hong, L., Chen, X. (2012). Vulnerability analysis of interdependent infrastructure systems: A methodological framework, Physica A: Statistical Mechanics and its Applications, Vol. 391, No. 11, 3323-3335, doi: 10.1016/j.physa.2011.12.043.

[10] Lei, T., Liangyu, W., Rijia, D., Lijia, L. (2011). Study on the dynamic input-output model with coal mine safety, Procedia Engineering, Vol. 26, 1997-2002, doi: 10.1016/i.proeng.2011.11.2396.

[11] Jiang, W., Deng, L., Chen, L., Wu, J., Li, J. (2009). Risk assessment and validation of flood disaster based on fuzzy mathematics, Progress in Natural Science, Vol. 19, No. 10, 1419-1425, doi: 10.1016/j.pnsc.2008.12.010.

[12] Miller, R.E., Blair, P.D. (2009). Input-output analysis: Foundations and extensions, (2 ${ }^{\text {nd }}$ edition), Cambridge University Press, New York, USA, doi: 10.1017/CB09780511626982.

[13] Jung, J., Santos, J.R., Haimes, Y.Y. (2009). International trade inoperability input-output model (IT-IIM): Theory and application, Risk Analysis, Vol. 29, No. 1, 137-154, doi: 10.1111/j.1539-6924.2008.01126.x.

[14] Tan, R.R., Aviso, K.B., Promentilla, M.A.B., Yu, K.D.S., Santos, J.R. (2015). Development of a fuzzy linear programming model for allocation of inoperability in economic sectors due to loss of natural resource inputs, DLSU Business \& Economics Review, Vol. 24, No. 2, 1-12.

[15] Tan, R.R., Aviso, K.B., Promentilla, M.A.B., Yu, K.D.S., Santos, J.R. (2014). Fuzzy inoperability input-output analysis of mandatory biodiesel blending programs: The Philippine case, Energy Procedia, Vol. 61, 45-48, doi: 10.1016/j.egypro.2014.11.902.

[16] Pant, R., Barker, K., Grant, F.H., Landers, T.L. (2011). Interdependent impacts of inoperability at multi-modal transportation container terminals, Transportation Research Part E: Logistics and Transportation Review, Vol. 47, No. 5, 722-737, doi: 10.1016/j.tre.2011.02.009.

[17] Resurreccion, J.Z., Santos, J.R. (2012). Stochastic modeling of manufacturing-based interdependent inventory for formulating sector prioritization strategies in reinforcing disaster preparedness, In: Proceedings of the 2012 IEEE Systems and Information Design Symposium (SIEDS), 134-139, doi: 10.1109/SIEDS.2012.6215127.

[18] Tan, R.R., Aviso, K.B., Promentilla, M.A.B., Solis, F.D.B., Yu, K.D.S., Santos, J.R. (2015). A shock absorption index for inoperability input-output models, Economic Systems Research, Vol. 27, No. 1, 43-59, doi: 10.1080/0953 5314. 2014.922462.

[19] Owusu, A., Mohamed, S., Anissimov, Y. (2010). Input-output impact risk propagation in critical infrastructure interdependency, In: Proceedings of the $13^{\text {th }}$ International Conference on Computing in Civil and Building Engineering, Nottingham, UK.

[20] Setola, R., De Porcellinis, S., Sforna, M. (2009). Critical infrastructure dependency assessment using the inputoutput inoperability model, International Journal of Critical Infrastructure Protection, Vol. 2, No. 4, 170-178, doi: 10.1016/j.ijcip.2009.09.002.

[21] Percoco, M. (2006). A note on the inoperability input-output model, Risk Analysis, Vol. 26, No. 3, 589-594, doi: 10.1111/j.1539-6924.2006.00765.x.

[22] Wei, H., Dong, M., Sun, S. (2010). Inoperability input-output modeling (IIM) of disruptions to supply chain networks, Systems Engineering, Vol. 13, No. 4, 324-339, doi: 10.1002/sys.20153.

[23] Barker, K., Santos, J.R. (2010). Measuring the efficacy of inventory with a dynamic input-output model, International Journal of Production Economics, Vol. 126, No. 1, 130-143, doi: 10.1016/j.ijpe.2009.08.011.

[24] Benjamin, M.F.D., Tan, R.R., Razon, L.F. (2015). Probabilistic multi-disruption risk analysis in bioenergy parks via physical input-output modeling and analytic hierarchy process, Sustainable Production and Consumption, Vol. 1, 22-33, doi: 10.1016/j.spc.2015.05.001.

[25] Garvey, P.R., Pinto, C.A., Santos, J.R. (2014). Modelling and measuring the operability of interdependent systems and systems of systems: advances in methods and applications, International Journal of System of Systems Engineering, Vol. 5, No. 1, 1-24, doi: 10.1504/IJSSE.2014.060880. 
[26] Percoco, M. (2011). On the local sensitivity analysis of the inoperability input-output model, Risk Analysis, Vol. 31, No. 7, 1038-1042, doi: 10.1111/i.1539-6924.2010.01574.x.

[27] Oliva, G., Panzieri, S., Setola, R. (2010). Agent-based input-output interdependency model, International Journal of Critical Infrastructure Protection, Vol. 3, No. 2, 76-82, doi: 10.1016/i.ijcip.2010.05.001.

[28] Crowther, K.G. (2008). Decentralized risk management for strategic preparedness of critical infrastructure through decomposition of the inoperability input-output model, International Journal of Critical Infrastructure Protection, Vol. 1, 53-67, doi: 10.1016/i.ijcip.2008.08.009.

[29] Hester, P.T., Adams, K.MacG. (2013). Determining stakeholder influence using input-output modeling, Procedia Computer Science, Vol. 20, 337-341, doi: 10.1016/i.procs.2013.09.282.

[30] Lian, C., Santos, J.R., Haimes, Y.Y. (2007). Extreme risk analysis of interdependent economic and infrastructure sectors, Risk Analysis, Vol. 27, No. 4, 1053-1064, doi: 10.1111/j.1539-6924.2007.00943.x.

[31] Xu, W., Wang, Z. (2015). The uncertainty and sensitivity analysis of the interdependent infrastructure sectors based on the supply-driven inoperability input-output model, International Journal of Innovative Computing, Information and Control, Vol. 11, No. 2, 615-625.

[32] Whiteman, H. (2014). Philippines gets more than its share of disasters, from http://edition.cnn.com/ 2013/11/08/world/asia/philippines-typhoon-destruction/,accessed January 4, 2016.

[33] Mondragon, A. (2013). Antique's agriculture still reeling from Yolanda, from http://www.rappler.com/moveph/46911-antique-agriculture-yolanda, accessed January 6, 2016.

[34] Olchondra, R.T., Abadilla, D.D. (2015). 5 dead, 4 missing in coal mine collapse. Inquirer News, from http://newsinfo.inquirer.net/705980/5-dead-4-missing-in-coal-mine-collapse, accessed January 6, 2016.

[35] Li, M., Wu, G.-D., Lai, X.D. (2014). Capacity coordination mechanism for supply chain under supply-demand uncertainty, International Journal of Simulation Modelling, Vol. 13, No. 3, 364-376, doi: 10.2507/IJSIMM13(3)C014.

[36] Seuring, S. (2013). A review of modeling approaches for sustainable supply chain management, Decision Support Systems, Vol. 54, No. 4, 1513-1520, doi: 10.1016/j.dss.2012.05.053.

[37] Smew, W., Young, P., Geraghty, J. (2013). Supply chain analysis using simulation, Gaussian process modelling and optimisation, International Journal of Simulation Modelling, Vol. 12, No. 3, 178-189, doi: 10.2507/IJSIMM12 (3) 4.239.

[38] Očevčić, H., Nenadić, K., Šolić, K. (2014). Decision support based on the risk assessment of information systems and Bayesian learning, Tehnički vjesnik - Technical Gazette, Vol. 21, No. 3, 539-544. 